\title{
BMJ Open Exploring definitions of radiological sarcopenia in cancer: a protocol for a scoping review
}

To cite: Wang JW, Williams M. Exploring definitions of radiological sarcopenia in cancer: a protocol for a scoping review. BMJ Open 2021;11:e053076. doi:10.1136/ bmjopen-2021-053076

- Prepublication history for this paper is available online To view these files, please visit the journal online (http://dx.doi org/10.1136/bmjopen-2021053076).

Received 04 May 2021 Accepted 17 June 2021

Check for updates

(C) Author(s) (or their employer(s)) 2021. Re-use permitted under CC BY-NC. No commercial re-use. See rights and permissions. Published by BMJ.

${ }^{1}$ Computational Oncology Laboratory, Imperial College London, London, UK

${ }^{2}$ Department of Radiotherapy, Charing Cross Hospital, London, UK

Correspondence to Dr James Wei Wang: james.wang@nhs.net

\section{ABSTRACT}

Introduction Sarcopenia is the loss of skeletal muscle volume or quality, a concept previously established in agerelated frailty. Sarcopenia is part of the cancer cachexia syndrome and has therefore been explored as biomarker through the opportunistic measurement of skeletal muscle from routine cancer imaging. However, there is inconsistency in diagnostic landmarks and cut-offs. The most common assessment method is skeletal muscle area at the slice level of the third lumbar vertebrae divided by height squared. Alternative sarcopenia measures have been derived from morphological descriptions of the psoas, thoracic and cervical muscles, driven by tumourspecific anatomical imaging.

Current tumour-site specific reviews suggest a link between heterogeneously defined sarcopenia on tumour site-specific outcomes. Because lack of uniformity, a scoping review is best suited to streamline anatomically based definitions and map the evidence to outcomes. The aim of this article is to describe a protocol for a scoping review that will homogenise the evidence of radiological sarcopenia in cancer. The extent, range and nature of reports will be examined, after which possible titles for potential systematic reviews identified.

Methods and analysis We will apply methods based on the Joanna Briggs Institute scoping review manual. Predefined search terms compiled with a librarian experienced in systematic reviews will be used to search PubMed/Medline, Embase, Scopus and Cochrane databases studies correlating cross-sectional cancer sarcopenia biomarkers with clinical outcomes. Studies will be mapped according to whether they have defined new sarcopenia measures or applied previous definitions to new populations, both with reported outcomes. This review will generate a numerical analysis on the extent of cancer sarcopenia measures as well as a narrative synthesis to describe the applications of radiologically derived sarcopenia in cancer.

Ethics and dissemination Formal ethical approval was not required to undertake this scoping review. Findings will be published in peer-reviewed journals and conference presentations.

\section{INTRODUCTION}

Sarcopenia was defined as loss of skeletal muscle volume or quality below $2 \mathrm{SD}$ of the population mean when introduced as a marker of frailty in the geriatric population. ${ }^{1}$

\section{Strengths and limitations of this study}

This protocol provides a comprehensive mapping strategy for the definitions of sarcopenia derived from cross-sectional imaging in cancer and how it has been applied in the literature.

- The review team includes experienced oncologists and the cross-examination of all searches with inter-rater cohesion will allow for a robust strategy.

- The search is limited to studies in English which may underestimate the quantity and type of sarcopenia markers found in non-English-speaking regions.

As a shared phenotype between age-related physiological loss and the cachexia syndrome, sarcopenia has also been adopted as a prognostic and predictive biomarker in cancer. Sarcopenia can be quantified functionally, radiologically or by bioelectrical impedance. While functional evaluations such as hand grip strength and bioelectric analysis are not routinely performed in cancer care, medical imaging is an integral part of cancer assessment. Cross-sectional imaging in the form of CT and MRI is used to quantify tumour burden within the body and is often repeated to monitor for treatment response and recurrence. They are also considered the gold standard in the non-invasive assessment of muscle quantity. ${ }^{2}$

Opportunistic measurement of skeletal muscle from routine imaging yields semantic measures of volume and quality. The primary objective in cancer sarcopenia studies is to either (1) find the optimal cut-off of low skeletal muscle parameters to predict a clinical end-point or (2) explore the validity of these endpoints in a separate population and/or endpoint. However, there is inconsistency in landmarks measured, diagnostic cut-offs, and the clinical outcomes used to justify the definitions. The most widely adopted approach of sarcopenia assessment is of the skeletal muscle index (cross-sectional area divided by the height squared) at the level of the third 
lumbar vertebrae (L3SMI) due to previous work relating it to whole body muscle mass. ${ }^{3}$ Alternative measures of sarcopenia have been derived from temporalis muscle area, psoas muscle area/index, thoracic muscle index and cervical muscle index, driven by the availability of tumour-specific anatomical imaging ${ }^{4-6}$ over the strength of their correlation with whole body muscle mass. A binary cut-off separating populations into sarcopenic versus non-sarcopenic allows for an easily applicable diagnostic label, but inherits the single institution biases present in the majority of studies.

The lack of consensus framework serves as a barrier to intervention and decreases prognostic value. ${ }^{7}$ In population-based studies on age-related sarcopenia, dual-energy X-ray absorptiometry (DXA) of limbs is the main radiological modality for muscle mass assessment. European, Asian and International consensus groups have published overlapping limb based DXA sarcopenia cut-offs of $7-7.23 \mathrm{~kg} / \mathrm{m}^{2}$ in men and $5.4-5.67 \mathrm{~kg} /$ $\mathrm{m}^{2} .{ }^{189}$ Similar values are recommended by a 2011 international consensus on cancer cachexia with 7.26/5.45 $\mathrm{kg} / \mathrm{m}^{2}(\mathrm{M} / \mathrm{F})$, which on conversion to L3SMI yields cutoffs of $<55 / 39 \mathrm{~cm}^{2} / \mathrm{m}^{2}(\mathrm{M} / \mathrm{F}) .{ }^{10}$ These normative values correlate with findings from western sarcopenia studies, but leads to discrepant findings in other demographics. In a cohort of Taiwanese pancreatic cancer patients, the prevalence of sarcopenia was inflated from $11 \%$ to $66.4 \%$ when applying cut-offs derived from a Japanese Hepatocellular Carcinoma study and a western study, respectively. ${ }^{11}$

Existing reviews on cancer sarcopenia focus on tumourtype specific outcomes, collating a mix of different methodological approaches and sarcopenia definitions. ${ }^{12} 13$ The most commonly reported end-point is overall survival (OS), but can include chemotherapy toxicity, surgical complications, disease-free survival (DFS) and length of stay. On the utility of sarcopenia metrics, a 2016 metaanalysis on L3SMI in cancer sarcopenia found cutoffs ranging between $29.6-41 \mathrm{~cm}^{2} / \mathrm{m}^{2}$ in women and $36-55.4 \mathrm{~cm}^{2} / \mathrm{m}^{2}$ in men among 38 studies. ${ }^{14}$ Although able to demonstrate a relationship with OS (HR 1.44, 95\% CI 1.32 to 1.56 ) despite the wide cut-off range, no clinically operational definition emerged.

It remains unclear if cancer sarcopenia is a concept more apparent in certain cancers or whether it is a universal syndrome analogous of cancer cachexia from which it stems. ${ }^{15}$ As a product of incidental biomarker generation from routine cancer imaging, operational definitions must respect these particular imaging modalities and the anatomy they capture if they are to be clinically applicable. A scoping review is therefore necessary to map the diversity of sarcopenia biomarkers and their impact on subsequent sarcopenia research. The full extent, range and nature of reports will allow for future systematic reviews and guidelines to then be considered.

The aim of this article is to describe a protocol for a scoping review to locate, map and summarise literature relating various definitions of cancer sarcopenia to cancer outcomes. The review seeks to answer the following question:

'How have sarcopenia measures in cancer crosssectional imaging been applied to clinical outcomes?'

The review will:

1. Identify cancer sarcopenia biomarkers have been defined by anatomical landmarks on routine crosssectional imaging.

2. Document how cancer sarcopenia definitions have been applied in the cancer sarcopenia research.

3. Assess the correlation of each sarcopenia definition in relation to reported outcomes.

\section{METHODS AND ANALYSIS \\ Study design}

This study protocol is based on the scoping review manuals provided by the Joanna Briggs Institute (JBI) ${ }^{16}$ A scoping review was chosen for its ability to map out the main sources and types of evidence available, allowing the synthesis of sarcopenia definitions by anatomical landmark.

\section{Search strategy}

The review will include original studies in English investigating cancer outcomes in relation to skeletal muscle mass assessed on cross-sectional image (CT and MRI). Studies utilising DXA will not be included as DXA, along with usual frailty-sarcopenia assessments are not part of routine cancer assessment and would therefore be difficult to apply clinically.

With assistance from a librarian experienced in conducting systematic searches, the review will search PubMed/MEDLINE, Embase, Scopus and Cochrane databases using the following search string: ("cancer" OR "carcinoma" OR "neoplasms" OR "chemotherapy" OR "radiotherapy" OR "chemoradiotherapy") AND ("sarcopenia" OR "skeletal" AND "muscle" OR "muscle" AND "index" OR "body" AND "mass" AND "index" OR "body" AND "composition" OR "muscle" AND "index" OR "atrophy") AND ("mri" or "ct). Exploded MESH or thesaurus terms will be used where possible to increase number of hits. No pre-specified anatomical landmarks have been included to avoid limitations from a priori knowledge. Studies will be imported on a reference manager for deduplication.

Title and abstract of documents retrieved from the bibliographic search will be screened by two independent reviewers before full texts are examined. The two reviewers will be actively practising oncologists with graduate-level oncology training. A preliminary analysis will be conducted on the first 100 results from one database search to ensure a high inter-rater reliability $(>0.8$ kappa score). The results of this preliminary analysis will inform any necessary protocol or definition changes that need to occur prior to proceeding or conducting a revised search. Title and abstract screening will be followed by full text retrieval and independent aggregation by two 
Table 1 PICOS design for identification of relevant studies for the scoping review

Population Inclusion: Human cancer patients aged 18+, any sex and stage of cancer. Cancer diagnosis confirmed according to diagnostic criteria relevant to the cancer group, which can combine histology, biochemistry and imaging.

Exclusion: Inclusion of non-cancer patients.

\begin{tabular}{|c|c|}
\hline Intervention & $\begin{array}{l}\text { Inclusion: Any sarcopenia assessment on cross-sectional MRI or CT. Although MRI and CT can cover any } \\
\text { anatomical landmark, the review will prioritise measures from imaging done during routine cancer imaging. } \\
\text { Exclusion: Routine cancer staging includes cranium, chest, abdomen and pelvis, meaning that limb-based } \\
\text { imaging will not be included. }\end{array}$ \\
\hline Comparison & $\begin{array}{l}\text { Inclusion: Non-sarcopenia cohort per the study's definition. } \\
\text { Exclusion: Studies without non-sarcopenia cohort (feasibility studies). }\end{array}$ \\
\hline Outcome & Survival, disease-free survival, treatment-related toxicity or complications, postoperative stays. \\
\hline Study Design & $\begin{array}{l}\text { Inclusion: Clinical trials or observational studies will be included } \\
\text { Exclusion: Case reports, abstracts, conference presentations, expert opinions, reviews and meta- } \\
\text { analyses. Where multiple studies share populations only the largest study will be included. }\end{array}$ \\
\hline
\end{tabular}

authors with conflicts resolved through arbitration by the lead author (JWW). Additional studies will be retrieved from reference sections of full text reviews.

\section{Identifying relevant studies}

Inclusion and exclusion criteria for title and abstract screening and full text articles will be based on the Population, Intervention, Comparator, Outcome and Study Design narrative are illustrated in table 1 . Studies are not limited by date but must have extractable data.

\section{Data extraction and charting}

The data will be charted onto a modified template from the JBI, collated onto spreadsheets. Two authors will pilot the chart data for 10 full text documents before training for data charting begins. A preliminary analysis will also be performed to pilot the data summary process.

Sarcopenia landmarks will be grouped into primary or secondary studies as represented in figure 1. Primary studies are defined as original studies which have generated de novo sarcopenia biomarker definitions, and secondary studies are those that have apply these landmarks to their own cohorts. Studies that do not identify the origin of their sarcopenia definition will be excluded. All the definitions will be extracted as reported, as no universal transformation exist to account for comparison between every possible candidate landmark. Sarcopenia definition methodology will be noted, for example, via receiver operation characteristic or division into tertiles.

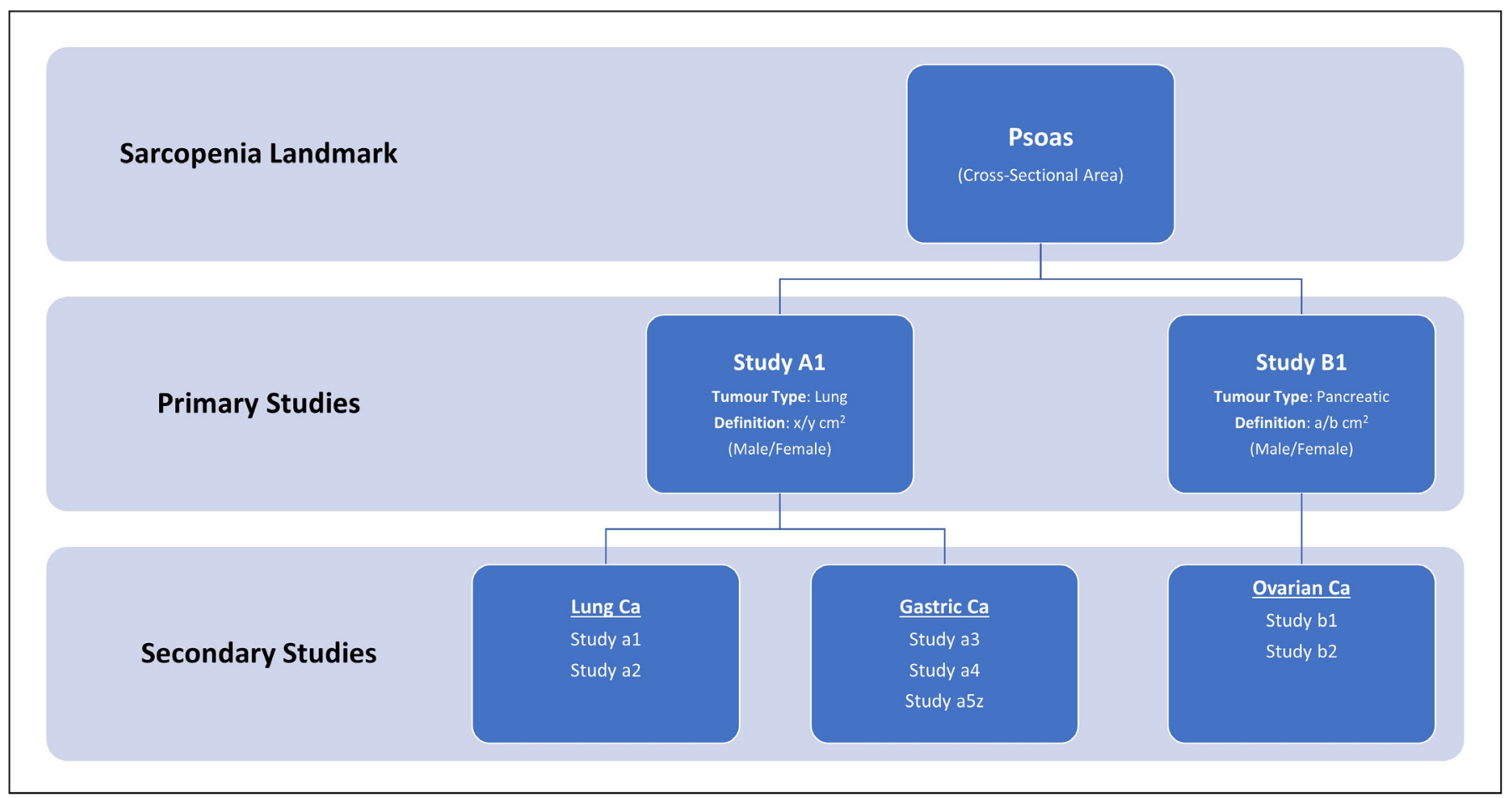

Figure 1 Concept map of sarcopenia studies by landmark definitions. 


\section{Box 1 Data extraction framework}

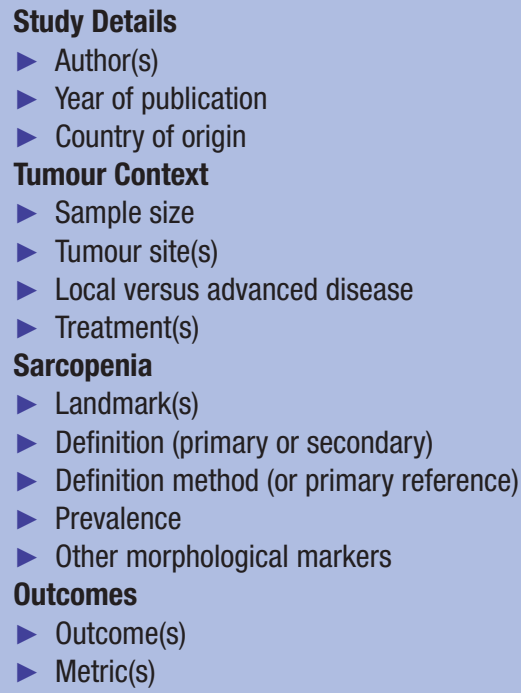

Outcomes of note will include survival and DFS (represented by HRs), length of stay (represented by medians) and treatment complications/toxicity (represented by ORs). Box 1 summarises the extraction framework. Associated $\mathrm{p}$ values will also be extracted when accompanied by regression or other analysis. This breakdown will allow for tracking of methodological biases as well as the proliferation and robustness of each individual sarcopenia landmark.

\section{Data presentation}

The review will use a variation of the synthesis without meta-analysis reporting guidelines. ${ }^{17}$ Data will be grouped by anatomical landmarks into concept maps, with subgroupings for variations on those landmarks where appropriate (eg, cross-sectional area vs skeletal index corrected for height). Within subgroupings, primary and secondary sarcopenia studies will be represented in hierarchical fashion using a graphical format such as a flow or spider diagram as demonstrated in figure 1 . Primary and secondary groupings will be summarised by tumour group and cancer stage. As the same landmarks are often applied across multiple tumour sites, this structure would allow narrative flow of sarcopenic descriptors have been created and their subsequent impact on the wider oncology literature. A numerical analysis will be performed on the extent, nature and distribution of reports included in the review in order to summarise the outcomes relating to the particular sarcopenia biomarker.

Studies will not be weighted numerically for the purposes of assigning priority, but rather will be represented by the impact of subsequent studies which use their definition and the significance of their primary outcome measure. No meta-analysis will be conducted as the purpose is to scope the definition. Instead, descriptive analysis of results will be summarised within each group in relation to charted data.

\section{Risk-of-bias assessment or quality appraisal}

As this is a scoping review aiming to map all available cancer sarcopenia landmarks and the propagation of such descriptors in subsequent studies, a risk-of-bias assessment will not be conducted. This is consistent with the JBI scoping review manual. ${ }^{16}$ For quality appraisal, we will prioritise studies by size and landmark derivation.

\section{Patient and public involvement}

The methodology in this protocol was informed by public and patient involvement focus group on sarcopenia on research held April 2021. Patients, families and carers contributed to the final decision on outcomes of interest from sarcopenia research.

\section{Ethics and dissemination}

Formal ethical approval was not required to undertake this scoping review. Findings will be published in peerreviewed scientific journals and presented in conferences.

Acknowledgements We wish to acknowledge the efforts of Kirsten Elliott of the Imperial College Library for her assistance in formulating search terms. This scoping review protocol is not further registered.

Contributors JWW conceptualised the study. MW and JWW designed the data extraction methodology, drawing from the Joanna Briggs Institute manuals. MW verified data extraction and JWW performed pilot run. JWW drafted the final version of protocol with supervision from MW.

Funding The authors have not declared a specific grant for this research from any funding agency in the public, commercial or not-for-profit sectors.

\section{Competing interests None declared.}

Patient and public involvement Patients and/or the public were involved in the design, or conduct, or reporting, or dissemination plans of this research. Refer to the Methods section for further details.

Patient consent for publication Not required.

Provenance and peer review Not commissioned; externally peer reviewed.

Open access This is an open access article distributed in accordance with the Creative Commons Attribution Non Commercial (CC BY-NC 4.0) license, which permits others to distribute, remix, adapt, build upon this work non-commercially, and license their derivative works on different terms, provided the original work is properly cited, appropriate credit is given, any changes made indicated, and the use is non-commercial. See: http://creativecommons.org/licenses/by-nc/4.0/.

ORCID iD

James Wei Wang http://orcid.org/0000-0002-1530-1055

\section{REFERENCES}

1 Cruz-Jentoft AJ, Bahat G, Bauer J, et al. Sarcopenia: revised European consensus on definition and diagnosis. Age Ageing 2019;48:16-31

2 Beaudart C, McCloskey E, Bruyère O, et al. Sarcopenia in daily practice: assessment and management. BMC Geriatr 2016;16:170.

3 Prado CMM, Lieffers JR, McCargar LJ, et al. Prevalence and clinical implications of sarcopenic obesity in patients with solid tumours of the respiratory and gastrointestinal tracts: a population-based study. Lancet Oncol 2008;9:629-35.

4 Jung H-W, Kim JW, Kim J-Y, et al. Effect of muscle mass on toxicity and survival in patients with colon cancer undergoing adjuvant chemotherapy. Support Care Cancer 2015;23:687-94.

5 Furtner J, Berghoff AS, Albtoush OM, et al. Survival prediction using temporal muscle thickness measurements on cranial magnetic resonance images in patients with newly diagnosed brain metastases. Eur Radiol 2017;27:3167-73.

6 Swartz JE, Pothen AJ, Wegner I, et al. Feasibility of using head and neck CT imaging to assess skeletal muscle mass in head and neck cancer patients. Oral Oncol 2016;62:28-33. 
7 Dunne RE, Loh KP, Williams GR. Cachexia and sarcopenia in older adults with cancer: a comprehensive review. Cancers 1861;2019:11.

8 Fielding RA, Vellas B, Evans WJ, et al. Sarcopenia: an undiagnosed condition in older adults. current consensus definition: prevalence, etiology, and consequences. International Working group on sarcopenia. J Am Med Dir Assoc 2011;12:249-56.

9 Chen L-K, Woo J, Assantachai P, et al. Asian Working group for sarcopenia: 2019 consensus update on sarcopenia diagnosis and treatment. J Am Med Dir Assoc 2020;21:300-7.

10 Fearon K, Strasser F, Anker SD, et al. Definition and classification of cancer cachexia: an international consensus. Lancet Oncol 2011;12:489-95.

11 Wu C-H, Chang M-C, Lyadov VK, et al. Comparing Western and eastern criteria for sarcopenia and their association with survival in patients with pancreatic cancer. Clin Nutr 2019;38:862-9.

12 Nishimura JM, Ansari AZ, D'Souza DM, et al. Computed Tomography-Assessed skeletal muscle mass as a predictor of outcomes in lung cancer surgery. Ann Thorac Surg 2019;108:1555-64.

13 Rossi F, Valdora F, Bignotti B, et al. Evaluation of body computed Tomography-determined sarcopenia in breast cancer patients and clinical outcomes: a systematic review. Cancer Treat Res Commun 2019;21:100154.

14 Shachar SS, Williams GR, Muss HB, et al. Prognostic value of sarcopenia in adults with solid tumours: a meta-analysis and systematic review. Eur J Cancer 2016;57:58-67.

15 Von Haehling S, Anker SD. Prevalence, incidence and clinical impact of cachexia: facts and numbers-update 2014. Wiley, 2014.

16 Peters M, Godfrey C, Mclnerney P. Chapter 11: Scoping Reviews. In: Aromataris E, Munn Z, eds. JBI manual for evidence synthesis, 2020.

17 Campbell M, McKenzie JE, Sowden A, et al. Synthesis without meta-analysis (swim) in systematic reviews: reporting guideline. BMJ 2020;72:16890 